\title{
Variations in first principles calculated defect energies in GaAs and their effect on practical predictions
}

\author{
DEEPAK*, D BALAMURUGAN ${ }^{\dagger}$ and K NANDI \\ Department of Materials and Metallurgical Engineering, ${ }^{\dagger}$ Department of Physics, Indian Institute of Technology, \\ Kanpur 208 016, India
}

\begin{abstract}
There is an abundant literature on calculations of formation and ionization energies of point defects in GaAs. Since most of these energies, especially the formation energies, are difficult to measure, the calculations are primary means of obtaining their values. However, based on the assumptions of the calculations, the reported values differ greatly among the various calculations. In this paper we discuss the sources of errors and their impact on practical predictions valuable in GaAs device fabrication. In particular, we have compared a large set of computed energies and selected the most appropriate values. Then, in the context of GaAs material quality, we investigated the impact of errors in calculation of formation energies on the performance of the GaAs substrate for device fabrication. We find that in spite of the errors inherent in $a b$ initio calculations, it is possible to correctly predict the behaviour of GaAs substrate.
\end{abstract}

Keywords. Ab initio calculations; semi-insulating GaAs; point defects.

\section{Introduction}

Theoretical study of native defects and dopants in solids can be carried out microscopically by electronic structure calculations. During last two decades, several such calculations have been done. We are focusing on gallium arsenide.

Jansen and Sankey (1989) used ab initio pseudo atomic orbital method to calculate relative point defect abundances in several III-IV and II-VI compound semiconductors. The pseudo-potentials, which were of Hamman, Schluter and Chiang type, are fit to a form prescribed by Bachelet et al (1982). The $s$ and $p$ atomic orbitals in ground state atomic configuration are used to construct the pseudo atomic orbitals. Similarly, Baraff and Schluter (1985) performed calculations on native point defects in GaAs by a Green's function scheme, using local density functional theory and first principles non-local pseudopotential of the same type as Jansen and Sankey. In both these calculations, however, relaxations were not included.

It is important to relax the ions while performing electronic structure calculations. There have been such theoretical studies using tight binding (TB) molecular dynamics (Seong and Lewis 1995) and $a b$ initio molecular dynamics, such as Car-Parrinello molecular dynamics (Northrup and Zhang 1993; Cheong and Chang 1994; Poykko et al 1996). The former method requires that the TB parameters are determined either empirically or using results from $a b$ initio methods. This is a disadvantage in comparison to the $a b$ initio methods, which are parameter

\footnotetext{
*Author for correspondence
}

free and, in principle, more reliable. However, the tight binding molecular dynamics is computationally less time consuming.

To investigate the point defects in GaAs, Seong and Lewis (1995) applied semi-empirical tight binding approach wherein the total energy includes the band structure energy, expressed in terms of a finite number of localized atomic orbitals, and a repulsive term, describing the ionic repulsion at short distances.

In comparison, more accurate calculations, at least in theory, for defect energetics that include structure relaxations are $a b$ initio molecular dynamics methods. Most of these are implemented using plane-wave pseudopotentials based on density functional theory. In this method, an extended system is simulated computationally with a supercell technique. The accuracy of the total energy calculated for the extended systems by this method sensitively depends on the number of plane waves, number of atoms in the supercell and the number of special $k$ points in the calculations.

In spite of similarity in the calculation technique, the calculated formation energies of native point defects of GaAs by Northrup and Zhang (1993), Cheong and Chang (1994) and Poykko et al (1996) differ in their values, although all of them employ plane wave pseudo-potential method within local density approximation. Northrup and Zhang calculated defect energies of native point defects of GaAs using scalar-relativistic pseudo-potential and a supercell containing 32 atoms. Kinetic energies up to 8 Ry were included in the plane wave expansion of the wave functions. Poykko et al used Bachelet-HammanSchluter type pseudo-potential and wave functions are expanded in a plane wave basis set corresponding to 
17 Ry energy cut-off. The calculations are with 64 atoms in the supercell. Cheong and Chang used a large plane wave cut-off corresponding to $30 \mathrm{Ry}$ with a supercell containing 32 atoms. They used Kleinman-Bylander type of fully separable pseudo-potential for calculating formation energy of native defects in GaAs and carbon dopant in it. In addition to plane wave cut-off criteria and the size of the system simulated in the supercell, the total energy calculation also depends on how the charge neutrality conditions in a supercell is maintained (Makov and Payne 1995; Schultz 2000).

A comparison of calculations of defect energies by these methods is provided in table 1. Since the estimate of ionization energies involves taking the difference of formation energy of a defect in the two charge states, we expect systematic errors to cancel out. Accordingly, we have compared only the ionization energies, rather than the defect formation energy in various charge states. The defects we consider are vacancies of $\mathrm{Ga}$ and $\mathrm{As}$, antisite defects and $\mathrm{Ga}$ and As interstitials. A missing entry in the table implies either the defect is predicted to be in only one charge state, that is, no ionization is possible, or no data is available.

The first two columns of data for ionization levels are based on the calculations where ionic relaxation has not been included. As discussed above, the remaining set of

Table 1. Comparison of ionization energies (measured from valence band edge).

\begin{tabular}{|c|c|c|c|c|c|c|c|}
\hline & \multirow{2}{*}{$\begin{array}{l}\text { Electronic } \\
\text { transition }\end{array}$} & \multicolumn{5}{|c|}{ Ionization levels $(\mathrm{eV})$} & \multirow[b]{2}{*}{$N \& Z^{a}$} \\
\hline & & $B \& S^{a}$ & $\mathrm{~J} \& \mathrm{~S}^{\mathrm{a}}$ & $S \& L^{a}$ & $C \& C^{a}$ & $\mathrm{P} e t a l^{\mathrm{a}}$ & \\
\hline \multirow[t]{5}{*}{$\mathrm{V}_{\mathrm{Ga}}$} & $+1 /-1$ & & & 0.035 & & & \\
\hline & $0 /-1$ & $0 \cdot 20$ & $0 \cdot 10$ & & & $0 \cdot 11$ & $0 \cdot 19$ \\
\hline & $-1 /-2$ & 0.50 & 0.35 & & 0.49 & $0 \cdot 22$ & $0 \cdot 20$ \\
\hline & $-2 /-3$ & 0.72 & 0.50 & & 0.69 & $0 \cdot 33$ & $0 \cdot 32$ \\
\hline & $-1 /-3$ & & & $0 \cdot 078$ & & & \\
\hline \multirow[t]{4}{*}{$\mathrm{V}_{\mathrm{As}}$} & $+2 /+1$ & $0 \cdot 10$ & & & & & \\
\hline & $+1 / 0$ & & $1 \cdot 30$ & 1.41 & & & \\
\hline & $0 /-1$ & & 1.40 & $1 \cdot 54$ & & & \\
\hline & $+1 /-1$ & & & & 0.785 & $0 \cdot 86$ & \\
\hline \multirow[t]{5}{*}{$\mathrm{Ga}_{\mathrm{As}}$} & $\begin{array}{l}+2 / 0 \\
+2 /+1\end{array}$ & & & 0.706 & & & \\
\hline & $+1 / 0$ & & & & & & \\
\hline & $0 /-1$ & $0 \cdot 30$ & $0 \cdot 10$ & & & & \\
\hline & $-1 /-2$ & 0.62 & $0 \cdot 35$ & & & & \\
\hline & $0 /-2$ & & & 0.896 & 0.695 & & $0 \cdot 38$ \\
\hline \multirow[t]{3}{*}{$\mathrm{As}_{\mathrm{Ga}}$} & $+2 /+1$ & $1 \cdot 25$ & 0.60 & & & & \\
\hline & $+1 / 0$ & 1.47 & 0.80 & & & & \\
\hline & $+2 / 0$ & & & 0.517 & $0 \cdot 70$ & & $0 \cdot 785$ \\
\hline \multirow[t]{2}{*}{$\mathrm{Ga}_{11}^{\mathrm{b}}$} & $+3 /+2$ & $0 \cdot 17$ & & & 0.72 & & 0.52 \\
\hline & $+2 /+1$ & 0.50 & $0 \cdot 10$ & & 0.89 & & 0.55 \\
\hline \multicolumn{2}{|l|}{$\mathrm{Ga}_{12}{ }^{\mathrm{b}}$} & & & & $\mathrm{c}$ & & \\
\hline \multirow[t]{5}{*}{$\mathrm{As}_{\mathrm{II}}^{\mathrm{b}}$} & $+3 / 0$ & & & & 0.93 & & \\
\hline & $+3 /+1$ & & & & & & 0.97 \\
\hline & $+3 /+2$ & $0 \cdot 80$ & $1 \cdot 25$ & & & & \\
\hline & $+2 /+1$ & $1 \cdot 13$ & 1.40 & & & & \\
\hline & $+1 / 0$ & & & & & & 1.05 \\
\hline \multirow[t]{3}{*}{$\mathrm{As}_{\mathrm{I} 2}{ }^{\mathrm{b}}$} & $+3 /+2$ & 0.60 & 0.80 & & & & \\
\hline & $+2 /+1$ & 1.05 & 0.95 & & $\mathrm{c}$ & & \\
\hline & $+1 / 0$ & $1 \cdot 30$ & $1 \cdot 10$ & & & & \\
\hline
\end{tabular}

${ }^{\mathrm{a}}$ B \& S: Baraff and Schluter (1985), J \& S: Jansen and Sankey (1989), S \& L: Seong and Lewis (1995), C \& C: Cheong and Chang (1994), P et al: Poykko et al (1996), N \& Z, Northrup and Zhang (1993).

${ }^{b}$ I1(I2): interstitial with 4 As (Ga) neighbouring atoms.

${ }^{\mathrm{c}} \mathrm{I} 1$ and $\mathrm{I} 2$ have not been distinguished. 
data consider relaxations in one form or another. In regard to gallium vacancies, S\&L (see table 1 for notation) finds a negative- $U$ effect, unlike in any other set of data. However, for most practical applications, where GaAs is either semi-insulating or $n$-type and Fermi energy is away from the valence band edge, the gallium vacancy in $\mathrm{S} \& \mathrm{~L}, \mathrm{P}$ et $a l$ and $\mathrm{N} \& \mathrm{Z}$ will exist in triple negative state; this state in $\mathrm{C} \& \mathrm{C}$ is reached only when Fermi energy is above $0.69 \mathrm{eV}$. Thus, based on the information that self-diffusion in GaAs occurs due to triply charged gallium vacancy, it would appear most accurate data is due to $\mathrm{P}$ et $a l$ and N\&Z, which in addition does not exhibit the negative $U$-effect.

$\mathrm{N} \& \mathrm{Z}$ predict that arsenic vacancy in GaAs to exist in only +1 charge state. This observation is consistent with that of $\mathrm{S} \& \mathrm{~L}$, where for almost entire range of Fermi energy in the band gap, most favourable charge state is +1 ; only near the conduction band edge, $1.41 \mathrm{eV}$ above the valence band, this charge state changes to neutral. In the other two cases, $\mathrm{C} \& \mathrm{C}$ and $\mathrm{P}$ et al, the ionization occurs in the middle of the band gap, inconsistent with the positron lifetime measurements (Seong and Lewis 1995).

Among the antisite defects, in all the calculations on gallium antisite defect that include relaxation, a negative U-effect is seen. However, this has not been measured experimentally. Furthermore, the calculated ionization level (0/-2) varies significantly between the various calculations. But, because generally the useful GaAs material is arsenic rich, this antisite defect may not play a role in determining the properties of the material. On the other hand, arsenic antisite defect is related to EL2 level in the middle of the band gap. The calculations due to $P$ et al and $\mathrm{N} \& \mathrm{Z}$ seem consistent with that observation.

Because the data provided by Northrup and Zhang (1993) is most comprehensive, seems most correlated with experimental observations and is based on an $a b$ initio method, which in principle is more accurate, we will regard this data as most useful for the subsequent use in determining properties of GaAs material.

In the remaining paper, we will show that data provided by Northrup and Zhang (1993) is capable of correctly predicting the electrical behaviour of GaAs. Furthermore, we will show that despite variations in the estimate of the formation energies of the defects, calculations for predicting the material behaviour are relatively insensitive to these errors.

\section{Results and discussion}

Using the data for formation energies of defects from Northrup and Zhang (1993), we have developed a detailed model for computation of point defect equilibrium. The model is based on considerations of several defect reactions. Solution of these equilibrium conditions along with the constraints of stoichiometry of the GaAs crystal and charge neutrality yields the relative predominance of the defects and also the concentration of free charge carriers. The details of the calculations are given elsewhere (Deepak and Lakshminarayana 2001; Deepak and Priyadarshi 2002).

\subsection{The model predictions}

The defect concentrations in a typical calculation are provided in figure 1 , where the carbon impurity is $1.3 \times 10^{16} \mathrm{~cm}^{-3}$ and the defect equilibrium establishes at $1200 \mathrm{~K}$. The constraint of a crystal stoichiometry is specified by $\alpha$ which is the atomic ratio of As and Ga. In the As rich region, as expected the most dominant defect is arsenic antisite, responsible for the semi-insulating (SI) behaviour because of the antisite's $+2 / 0$ donor level in middle of the band gap. Whereas, a Ga rich substrate shows hole conductivity (Holmes et al 1982) because as a relatively shallow acceptor, the $\mathrm{Ga}$ antisite, is most dominant. The most interesting feature is the sharp transition from $p$-type to semi-insulating behaviour. Also, realizing that a modern SI-GaAs wafer has EL2 (related to arsenic antisite) on order of $10^{16} \mathrm{~cm}^{-3}$, the stoichiometry of the crystals in use must lie in the sharp transition region. This introduces a possibility that a relatively small loss of As from the surface of the substrate during boule or wafer processing could lead to conducting $p$ type region. In turn, for example, this may result into spurious $p-n$ junction.

In order to test the prediction using the $a b$ initio computed data for the formation energies of the defects, we compare the measured free carrier concentrations provided by Holmes et al (1982) with our calculations. The

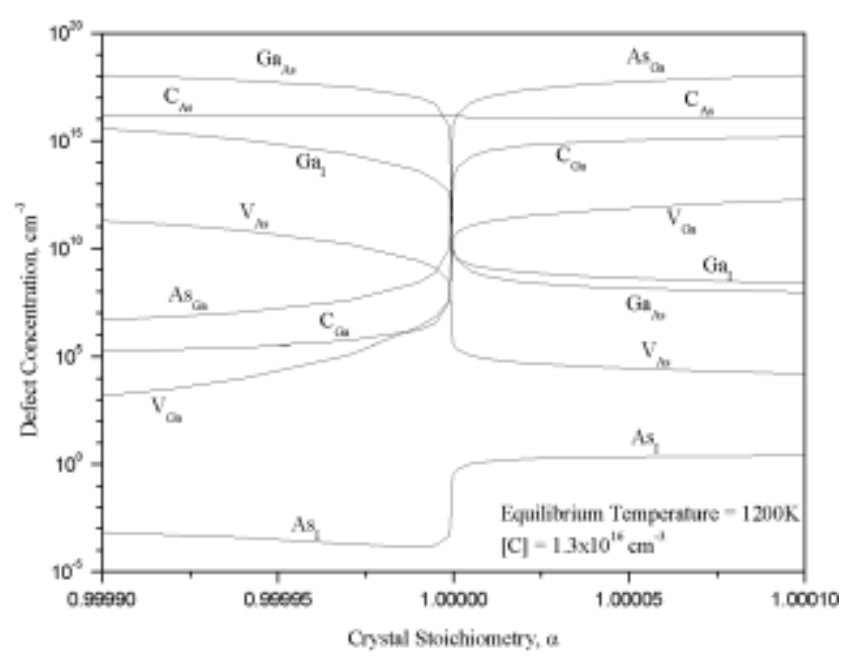

Figure 1. Relative predominance of defects in GaAs calculated by a thermodynamic model using ab initio calculated defect formation energies. 
computed results in figure 2 are for defect equilibrium at temperatures between $500 \mathrm{~K}$ and $1200 \mathrm{~K}$. In the calculations the equilibrium establishes at these temperatures, but then the material cools down rapidly to $300 \mathrm{~K}$ such that the total number of a particular kind of defect remains frozen corresponding to the higher equilibrium temperature and the charge states redistribute at $300 \mathrm{~K}$. Accordingly, we find the carrier concentrations are relatively insensitive to the defect equilibrium temperature. The measured data (Holmes et al 1982) compares well with the model predictions.

However, the modern SI substrates have an order of magnitude lower carbon impurity level $\left(10^{15} \mathrm{~cm}^{-3}\right)$. For this case of a lower carbon level, the free carrier concentration illustrated in figure 3 is also only weakly dependent on defect equilibrium temperature, mainly on the Ga rich side. However, the devices are made on As rich SI substrate, where the free carrier concentration is almost the same irrespective of the equilibrium temperature.

In short, the data for the formation energy, computed by an $a b$ initio method, is sufficiently reliable for describing the behaviour of GaAs material. However, we have also investigated the effect of possible variations/ errors in the formation energy data on the prediction of the material properties.

\subsection{Insensitivity to variations in defect formation energies}

In figure 3, the carrier concentration increases sharply after a sudden drop corresponding to the $p$-type to SI transition. Also, the sharpness of rise in carrier concentration in As rich region is dependent on the carbon impurity level (compare figure 2 with figure 3); the increase is more gradual at a higher impurity level.

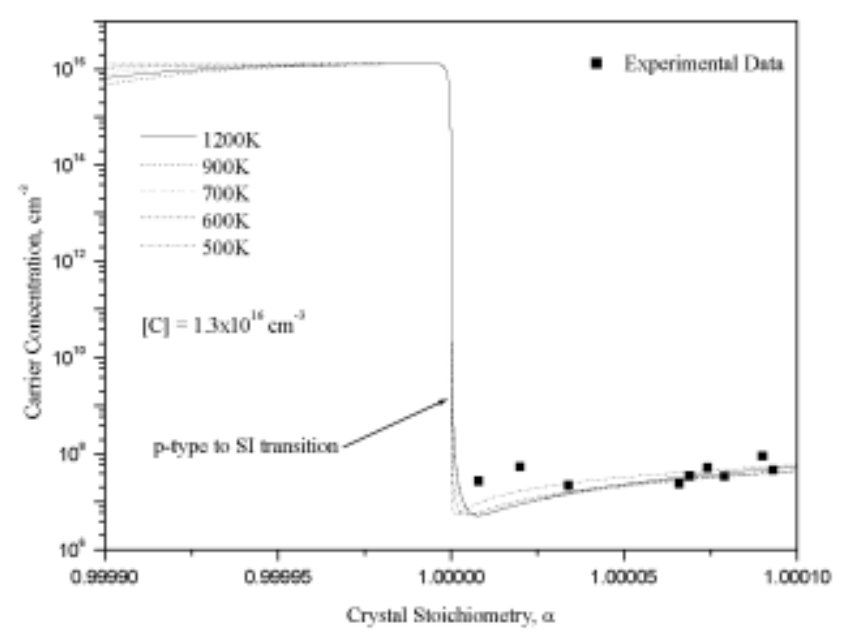

Figure 2. Calculated carrier concentrations are compared with experimental data. The first principles calculated defect energies are adequate for prediction of the material property.
A practical implication of this increase in carrier concentration in a commonly used semi-insulating GaAs material with carbon, $10^{15} \mathrm{~cm}^{-3}$, is variability in performance of the fabricated devices. For a material with arsenic antisite at $\sim 10^{16} \mathrm{~cm}^{-3}$ (which is common), the expected stoichiometry $(\infty)$ is 1.000001 . This stoichiometry lies in the region where the carrier concentration rises sharply after the $p$-type to SI transition. This means that a small variation in material stoichiometry could substantially change the conductivity, and in a worst case, two materials of same conductivity and almost same stoichiometry can have a significant difference in arsenic antisite level. This particular defect is known to have a major impact on dopant activation and hence device performance. Thus, potential variation in stoichiometry during growth of GaAs boule or As loss during processing has severe implications.

Therefore, we have selected this region in figure 3, where the carrier concentration increases rapidly after the sudden decrease, for investigating the effect of potential errors/variation in formation energies on practical prediction of the material properties. We allow a $\pm 15 \%$ variation in the formation energy of point defects in their lowest charge state (in effect, formation energy of the same defect type in all charge states changes by an equal amount), and then examine if this critical feature in the behaviour of carrier concentration is still predicted correctly.

For this purpose, our calculations are for defect equilibrium at $700 \mathrm{~K}$. In figure 3 , we show that the increase in carrier concentration after the $p$-type to SI transition is from mid- $10^{6}$ to mid- $10^{7} \mathrm{~cm}^{-3}$. Therefore, only those defects that have concentrations greater than this range can play a role in affecting the feature related to decrease and increase of the carrier concentration. Apart from the

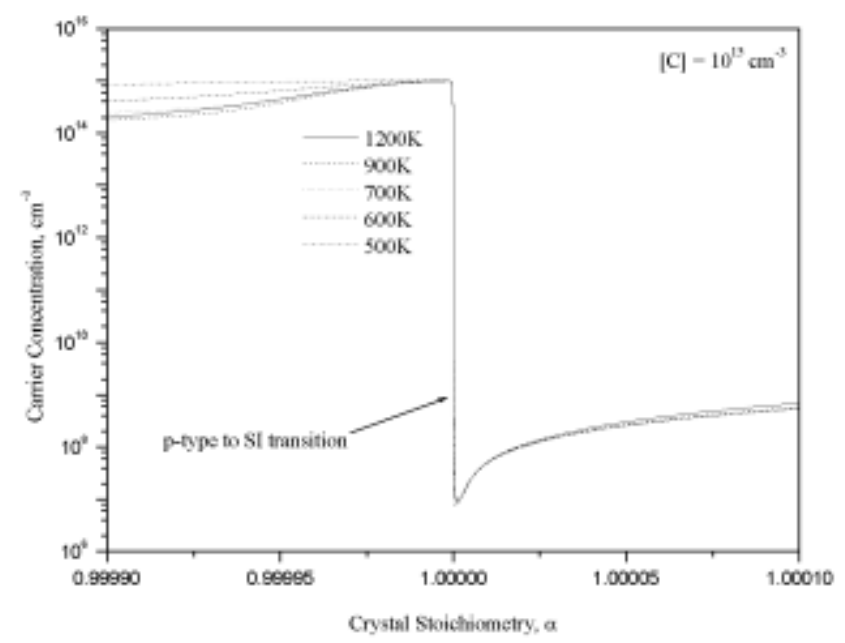

Figure 3. Carrier concentrations in a typical (carbon $10^{15} \mathrm{~cm}^{-3}$ ) GaAs substrate. The carrier concentration rises sharply following $p$-type to semi-insulating transition. 
carbon related defects (carbon on gallium and arsenic sites), only two additional defects, $\mathrm{As}_{\mathrm{Ga}}$ and $\mathrm{V}_{\mathrm{Ga}}$, are present in sufficient numbers. Therefore, only the defect formation energies of arsenic antisite $\left(\mathrm{As}_{\mathrm{Ga}}\right)$, carbon on arsenic site $\left(\mathrm{C}_{\mathrm{As}}\right)$, carbon on gallium site $\left(\mathrm{C}_{\mathrm{Ga}}\right)$ and vacancy of gallium $\left(\mathrm{V}_{\mathrm{Ga}}\right)$ are examined further; all other defects are in much lower concentrations.

Our initial calculation for stoichiometric GaAs and at two additional stoichiometries in slightly As-rich region reveals that a $15 \%$ decrease in the formation energy of arsenic antisite defect has no effect on the carrier concentration. This implies that an underestimate in the formation energy of arsenic antisite is unlikely to affect the presence of the feature in figure 3. However, an increase in the arsenic antisite formation energy has an enormous impact on the calculations of the carrier concentrations. This aspect is addressed in a greater detail subsequently.

Similarly, an increase in the defect formation energies of $\mathrm{V}_{\mathrm{Ga}}$ and $\mathrm{C}_{\mathrm{Ga}}$ and a decrease in that of $\mathrm{C}_{\mathrm{As}}$ have no impact on carrier concentrations. Conversely, a decrease in the defect formation energies of $\mathrm{V}_{\mathrm{Ga}}$ and $\mathrm{C}_{\mathrm{Ga}}$ and an increase in that of $\mathrm{C}_{\mathrm{As}}$ change the carrier concentrations, although only by a small amount. Therefore, the effect of formation energy increase for $\mathrm{As}_{\mathrm{Ga}}$ and $\mathrm{C}_{\mathrm{As}}$ and decrease for $\mathrm{V}_{\mathrm{Ga}}$ and $\mathrm{C}_{\mathrm{Ga}}$ is further evaluated.

The solid line in figure 4 represents the carrier concentrations for nominal values of formation energies, provided by Northrup and Zhang (1993). The crystal stoichiometry is varied in a small range in As rich region where the rapid changes in carrier concentrations are seen. Clearly, even after a $15 \%$ decrease in formation energy of $\mathrm{C}_{\mathrm{Ga}}$ and $\mathrm{V}_{\mathrm{Ga}}$, and a similar increase in that of $\mathrm{C}_{\mathrm{As}}$, the predicted feature involving the decrease and subsequent increase in carrier concentration is still present.

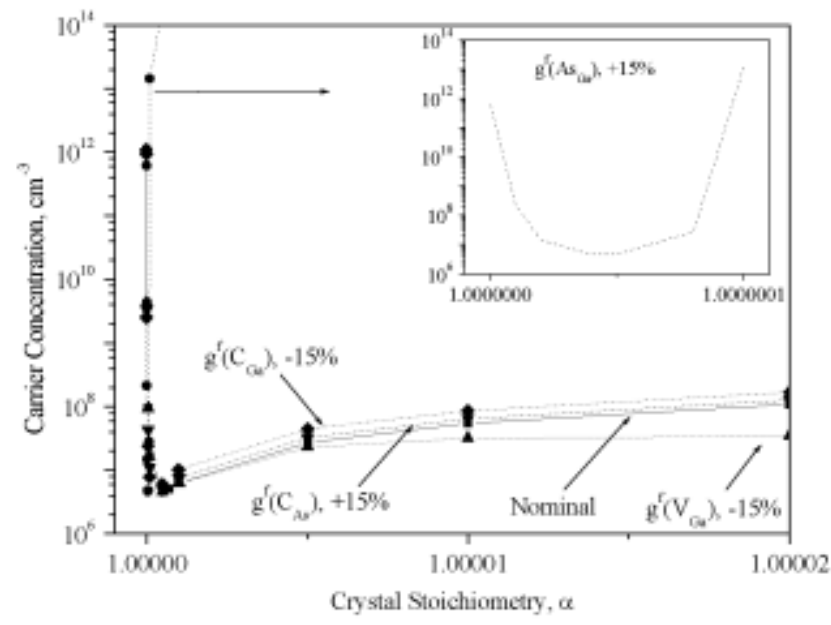

Figure 4. Effect of variation in formation energy of defects. The data markers are calculation points. The effect of change in formation energy of arsenic antisite is large and shown in the inset.
However, when the formation energy of the arsenic antisite is increased by $15 \%$, an unrealistic increase in carrier concentration is calculated (see inset in figure 4). Now the decrease and subsequent increase in carrier concentration is even more rapid. Thus, presence of this feature is still predicted, but the carrier concentrations in the As rich region become too large (n-type), while we experimentally know that material should be semi-insulating with carrier concentration on the order of $10^{7} \mathrm{~cm}^{-3}$.

Thus, we find that the practical prediction using these formation energies are insensitive to possible errors in their estimate for most of the defects; however, an accuracy better than $15 \%$ will have to be ensured for arsenic antisite.

\section{Conclusions}

A number of first principles studies on point defects in GaAs have been conducted. Specifically, we have reviewed the available data for the formation energies of point defects in GaAs. Based on the errors associated with the calculations and some experimental observations, the data provided by Northrup and Zhang (1993) appears most accurate and suitable.

Based on this data for formation energies, we have shown that an adequate estimate of material property, the carrier concentration in this work, can be made. These estimates are possible even when errors may be present in the calculations for the defect formation energies, which are parameters in the model for predicting the material properties. We also find, in large part, the predictions of the model are insensitive to the errors in $a b$ initio calculated formation energies of defects. The only defect whose value must be more accurate than $15 \%$ error is arsenic antisite.

However, when the practical predictions are guided by experimental measurements, it is possible to isolate the errors in formation energies and, in turn, thus it would be possible to improve on them.

\section{References}

Bachelet G B, Hamann D R and Schluter M 1982 Phys. Rev. B26 4199

Baraff G A and Schluter M 1985 Phys. Rev. Lett. 551327

Cheong B-H and Chang K J 1994 Phys. Rev. B49 17436

Deepak and Lakshminarayana N 2001 Bull. Mater. Sci. 24225

Deepak and Priyadarshi A 2002 Mater. Sci. Eng. B94 21

Holmes D E, Chen R T, Elliot K R, Kirkpatrick C G and Yu P W 1982 IEEE Trans. Microwave Theory and Tech. MTT-30 949

Jansen R W and Sankey O F 1989 Phys. Rev. B39 3192

Makov G and Payne M C 1995 Phys. Rev. B51 4014

Northrup J E and Zhang S B 1993 Phys. Rev. B47 6791

Poykko S, Puska M J and Nieminen R M 1996 Phys. Rev. 353 3813

Schultz P A 2000 Phys. Rev. Lett. 841942

Seong H and Lewis L J 1995 Phys. Rev. B52 5675 